\title{
Analysis of the effects of diversification for Dar es Salaam stock exchange optimal portfolio
}

\author{
Phares Kaboneka ${ }^{1,}$, Wilson Mahera Charles ${ }^{1,2}$, Silas Mirau ${ }^{1}$ \\ ${ }^{1}$ Department of Applied Mathematics and Computational Science and Engineering, Nelson Mandela African Institution of Science and \\ Technology, Arusha, Tanzania \\ ${ }^{2}$ Department of mathematics, University of Dar Es Salaam (UDSM), Dar Es Salaam, Tanzania
}

\section{Email address:}

kabonekap@nm-aist.ac.tz (P. Kaboneka), maheraii@yahoo.com (W. C. Mahera), silas.mirau@nm-aist.ac.tz (S. Mirau)

\section{To cite this article:}

Phares Kaboneka, Wilson Mahera Charles, Silas Mirau. Analysis of the Effects of Diversification for Dar Es Salaam Stock Exchange Optimal Portfolio. Applied and Computational Mathematics. Vol. 3, No. 5, 2014, pp. 205-216. doi: 10.11648/j.acm.20140305.13

\begin{abstract}
Dar es Salaam stock exchange (DSE) market is among the stock markets dealing with financial securities transactions and it operates under the brokerage system. Different individuals have little knowledge on how these stock markets operate and many of them fear to invest in stock business because they don't have the base line of their decision especially on the risk bearings. This paper is based solely on DSE stocks data for the period of past nine years and it tries to give out the nature of return of the stocks, the effects on restrictions at the DSE stock environment to the stock returns and also it explores the effect of diversification on return and on risk (standard deviation). The study uses the classical Markowitz Modern Portfolio Theory (MPT) model in its analysis with little modification so as to meet with the DSE environment. Data from DSE was analysed by using the excel solver and its macros like the solver add - in. After the analysis it is observed that restrictions have an effect on the stock risk and return, where it reduce risk and increases return because the unconstrained frontier is greater than the constrained frontier. Moreover it is found that for the diversification to have a significant effect the stocks have to be nearly or perfectly negatively correlated.
\end{abstract}

Keywords: Stocks, Diversification, DSE, Frontier, Covariance Matrix, Expected Return, Portfolio

\section{Introduction}

Financial status of any company or institution is not static. It changes over time due to a number of factors that can affect its financial flow. Among the factors that affects the financial flow includes inflation, decrease in human consumption rate caused by inflation, increase in investments, price fluctuation and government policy. Due to these factors we can easily see that financial and insurance markets always operate under various types of uncertainties that can affect financial positions of companies and individuals. In financial and insurance theories these uncertainties are usually referred to as risks. In stock markets where certificates of partial ownership of business are traded so as to raise the initial capital of the company for operation aspects, the stock market remains the major means of investment and can be used as an indicator of overall economic health. Due to this reason different countries have their own stock markets where prices are determined by the forces of demand and supply. Given certain states of the market, and the economy in general, one can talk about risk exposure. Any economic activities of individuals, companies and public establishments aiming for wealth accumulation assume studying risk exposure is of great importance [1]

Governments own companies or have shares on different companies that acts as the financial security in case of financial crisis. The shareholder becomes part of the company ownership. During the financial crisis the shareholder can sell the shares he owns. The shares are sold at the stock exchange markets [2]

In a business, Investors have to find the best way to price their business that will have the minimum risk in time. Because different investments have different turnover over time due to changing of the sales caused by customer consumption and investment rate, one will need to have the financial flow throughout the year by investing in both of the two portfolios [3]

Trade decisions are more concerned with the speed, costs, and risks associated with executing the transaction, while 
investment emphasizes selection of the security. To be able to make a good decision one needs to have a good knowledge on the pricing theory and pricing model. There are many models formulated that aims to reduce risk and maximize profit by providing a frame work for portfolio selection that have a minimum risk in time and among them is the Modern portfolio theory (MPT). It is used in pricing assets so as to have the minimum risk and gain the maximum return. Although it is more useful it does not operate in the same way in all the stock markets due to markets difference, and market governing rules [4]

This study aims at examining the DSE stock returns and explores the effects of diversification and restrictions on its environment so as to provide a frame work to the DSE investors on how they can invest in various stocks with a minimum risk level.

\section{Literature Review}

Portfolio allocation is independent for each individual investor basing upon several factors, including age, investable years before retirement, risk, required necessary return, and current or future goals. The overall positioning of a portfolio is important in evaluating the portfolios. A goal in any portfolio is to achieve the greatest amount of return while taking the least amount of risk. Also each security has its own deviation from an expected return statistically known as standard deviation from the mean in finances it is called risk. The risk of an overall portfolio is expected to decrease as the number of securities increase. According to Markowitz it is not only about pricing securities, but it is about choosing the most appropriate allocation of securities because different securities brings in different concepts of risk. An investor seeking higher return he also intrinsically take an increased risk because return to risk ratio grows quickly at first with each unit of additional risk eventually brings less and less opportunity for the return. This implies that for an investor to benefit more he has to get the optimal portfolio that has minimum risk and brings the expected return to do this one have to diversify his portfolio [5],[6]

Markowitz suggested that if we treat single-period returns for various securities as random variables, we can assign them expected values, standard deviations and correlations. And we can calculate the expected return and volatility of any portfolio constructed with those securities. Out of the entire universe of possible portfolios, we are sure that there is ones that will optimally balance risk and return. These comprise what Markowitz called an efficient frontier of portfolios. An investor should select a portfolio that lies on the efficient frontier.

The foundations of MPT resulted to the establishment of a formal risk-return framework for investment decision-making. By defining investment risk in quantitative terms, Markowitz gave investors a mathematical approach to asset selection and portfolio management by considering the mean and variances of portfolios. However, differing to its theoretical reputation, the mean- variance model has not been used extensively in its original form to construct a large-scale portfolio, it is computationally difficulty and it is associated with solving a large-scale quadratic programming problem with a dense covariance matrix. Several authors tried to alleviate this difficulty by using various approximation schemes Sharpe, [7], [8] in the early years of the history.

To force the portfolio to the efficient frontier [9] added a risk-free asset to the analysis and hence bringing up the concept of super-efficient portfolio and the capital market line. The capital asset pricing model (CAPM) makes strong assumptions that lead to interesting conclusions [10] Not only does the market portfolio sit on the efficient frontier, but it is actually Tobin's super-efficient portfolio [11] proposed a new portfolio optimization model using piecewise linear risk functions showing that their model can achieve the intention of Markowitz by solving a linear program instead of a difficult quadratic program and they emphasize on the use of LI risk (absolute deviation) model that leads to a linear program instead of a quadratic program, so that a large-scale optimization problem of more than 1,000 stocks may be solved on a real time basis. Various aspects of this phenomenon have been extensively studied in the literature on portfolio selection.

All these readings show that portfolio optimization is rooted from the Markowitz mean-variance model (MPT-model) and all the other models are directly or indirectly based on this model. However it is important to note that these models have been tested and used in few developed financial markets like the New York Stock Exchange (NYSE), most research papers analysis has been done using yahoo finance data, with some stocks like the S \& $\mathrm{P}$ '100, Nikkei, FTSE 100' appearing in most of the papers, [12] while different Financial Markets have different characteristics and their securities behave differently Konno and [13] its effectiveness in other markets is not guaranteed, showing a need to examine the applicability of these portfolio optimization models before implementing them in the budding stock markets like DSE.

Therefore this study aims to develop a model that will be adapted to the Dar es Salaam Stock Exchange market, basing on Modern Portfolio Theory. And the contribution of this study will be to explore the relevance and applicability of the modern portfolio theory model to the Dar es Salaam Stock Market as far as portfolio optimization is concerned.

\section{Materials and Methods}

\subsection{Model Formulation and Model Equations}

The methodology employed is to adopt the Markowitz model and simulate it with the data obtained from the Dar es Salaam stock of exchange market so as to come up with the optimal portfolio through solving the expectations and volatility of the portfolios.

Investors aim to maximize profit in the future context which is not sure and it must be anticipated or expected. Because it 
follows on these grounds it follows the normal probability theories of predictions and for the investor to have higher probability of getting profit has to invest in the portfolio with high return and low risk.

In the Markowitz mean - variance portfolio theory the rate of return on assets are assumed to be random variables. And the goal is to choose the portfolio with optimal weighting factor and which in the Markowitz context is the one with acceptable expected baseline.

The yield of the portfolio as a whole will be found by using the formula $R=\sum_{i} R_{i} X_{i}$

\subsection{Mean Variance Analysis}

Mean variance analysis is the core of MPT. For any two assets the mean of the portfolio is given by $\mu_{p}=\sum_{i=1}^{n} X_{i} \mu_{i}$ where $\mu_{i}$ is the mean $\left(\overline{R_{i}}\right)$ of the $R_{i} \forall_{\mathrm{i}=1,2,3, \ldots \mathrm{n}}$ and the variance is given by

$$
\sigma_{p}^{2}=\sum_{i=1}^{n} \sum_{j=1}^{n} \sigma_{i j} X_{i} X_{j}
$$

$\mu_{p}$ is the desired level of expected return

$\sigma_{p}^{2}$ is the desired level of variance

Reference [19] the covariance of $R_{i}$ and $R_{j}$ is denoted as $\operatorname{Cov}\left(R_{i}, R_{j}\right)$ or $\sigma_{i j}$ and is defined by the equation

$$
\sigma_{i j}=E\left[R_{i}-E\left(R_{i}\right)\right]\left[R_{j}-E\left(R_{j}\right)\right]
$$

Now letting $\mathrm{V}$ to denote a covariance matrix so that

$$
\sigma_{p}^{2}=X^{T} V X \text { where } X=\left(X_{1} X_{2}\right)^{T}
$$

From $\sum_{i=1}^{n} X_{i}=1$ (the sum of the weights of in the portfolio is $100 \%$ ) we get $X_{1}=1-\alpha$ and the equation $\mu_{p}=\sum_{i=1}^{2} X_{i} \mu_{i}$ becomes $\mu_{p}=\overline{R_{p}}=(1-\alpha) \overline{R_{1}}+\alpha \overline{R_{p}}$ where $0 \leq \alpha \leq 1$ and $X_{1}^{2} \sigma_{1}^{2}+X_{1} X_{2}\left(\begin{array}{ll}\sigma_{12} & \sigma_{21}\end{array}\right)+X_{2}^{2} \sigma_{2}^{2}$ becomes

$$
\sigma_{p}^{2}=(1-\alpha)^{2} \sigma_{1}^{2}+2 \rho \alpha(1-\alpha) \sigma_{1} \sigma_{2}+\alpha^{2} \sigma_{2}^{2}
$$

Hence the model for the two risk assets becomes

$$
\begin{gathered}
\overline{R_{p}}=(1-\alpha) \overline{R_{1}}+\alpha \overline{R_{2}} \text { for } 0 \leq \alpha \leq 1 \\
\sigma_{p}^{2}=(1-\alpha)^{2} \sigma_{1}^{2}+2 \rho \alpha(1-\alpha) \sigma_{1} \sigma_{2}+\alpha^{2} \sigma_{2}^{2} \text { for } 0 \leq \alpha \leq 1
\end{gathered}
$$

Then we have to solve these two equations for different values of $\alpha$ in the interval $-1 \leq \alpha \leq 1$

\subsection{Solution to the Two Asset Model Equation}

From equation (4) if $\alpha=1$ we get $\sigma_{p}^{2}=(1-\alpha)^{2} \sigma_{1}^{2}+2 \alpha(1-\alpha) \sigma_{1} \sigma_{2}+\alpha^{2} \sigma_{2}^{2} \quad$ which after solving results to $\sigma_{p}=(1-\alpha) \sigma_{1}+\alpha \sigma_{2}$

From this if $\alpha=0$ and 1 respectively we get $\sigma_{p}=\sigma_{1}$ and $\sigma_{p}=\sigma_{2}$

Again substituting the value of $\alpha=0$ and $\alpha=1$ in the first equation we get

$\overline{R_{p}}=\overline{R_{1}}$ for $\alpha=0$ and $\overline{R_{p}}=\overline{R_{2}}$ for $\alpha=1$ this implies that for $\alpha=0$ the portfolio value is $P_{0}\left(\begin{array}{ll}\sigma_{1} & \overline{R_{1}}\end{array}\right)$ to mean all the investments are in 1 stock and for $\alpha=1$ the portfolio value is $P_{1}\left(\begin{array}{ll}\sigma_{2} & \overline{R_{2}}\end{array}\right)$ to mean all the investments are in the second stock. Since $-1 \leq \rho \leq 1$ now substituting $\rho=-1$ into the

$\sigma_{p}^{2}=(1-\alpha)^{2} \sigma_{1}^{2}+2 \alpha \rho(1-\alpha) \sigma_{1} \sigma_{2}+\alpha^{2} \sigma_{2}^{2} \quad$ We get $\sigma_{p}^{2}=(1-\alpha)^{2} \sigma_{1}^{2}+2 \alpha(1-\alpha) \sigma_{1} \sigma_{2}+\alpha^{2} \sigma_{2}^{2}$ after solving we get $\sigma_{p}=\left|(1-\alpha) \sigma_{1}-\alpha \sigma_{2}\right|$ and for $\alpha=0$ we get the point $P_{0}\left(\begin{array}{ll}\sigma_{1} & \overline{R_{1}}\end{array}\right)$ and when $\sigma_{p}=0$ i.e $(1-\alpha) \sigma_{1}-\alpha \sigma_{2}=0$ solving for $\alpha$ from this equation we get $\alpha=\frac{\sigma_{1}}{\sigma_{1}+\sigma_{2}}$ and $\overline{R_{p}}=\left[1-\frac{\sigma_{1}}{\sigma_{1}+\sigma_{2}}\right] \overline{R_{1}}+\left(\frac{\sigma_{1}}{\sigma_{1}+\sigma_{2}}\right) \overline{R_{p}}$

Hence the portfolio value becomes $A\left(0,\left(1-\frac{\sigma_{1}}{\sigma_{1}+\sigma_{2}}\right) \overline{R_{1}}+\left(\frac{\sigma_{1}}{\sigma_{1}+\sigma_{2}}\right) \overline{R_{p}}\right)$ when plotted in the graph it will be easily seen that the point $\mathrm{Ap}_{\mathrm{o}}$ corresponds to the equation $\sigma_{p}=(1-\alpha) \sigma_{1}-\alpha \sigma_{2}$ and we have to note that the value of $(1-\alpha) \sigma_{1}-\alpha \sigma_{2}$ remain positive until when $\alpha=\frac{\sigma_{1}}{\sigma_{1}+\sigma_{2}} \quad$ and when $\quad \alpha>\frac{\sigma_{1}}{\sigma_{1}+\sigma_{2}} \quad$ the quantity $(1-\alpha) \sigma_{1}-\alpha \sigma_{2}$ becomes negative leading to $(\alpha-1) \sigma_{1}+\alpha \sigma_{2}$ and as $\alpha$ approaches to $1, \sigma_{1}=\sigma_{2}$ which gives the corresponding point close to $P_{1}\left(\sigma_{2} \overline{R_{2}}\right)$ and the locus will trace out the line $\mathrm{AP}_{1}$

Lastly we have to consider the interval $-1<\rho<1$ (this is the case of real data as those that will be obtained at DSE)

The minimum variance point are obtained by solving the equation $\frac{\partial \sigma_{p}^{2}}{\partial \alpha}=0$ 


$$
\begin{aligned}
& \frac{\partial \sigma_{p}^{2}}{\partial \alpha}=\frac{\partial}{\partial \alpha}\left((1-\alpha)^{2} \sigma_{1}^{2}+2 \alpha \rho(1-\alpha) \sigma_{1} \sigma_{2}+\alpha^{2} \sigma_{2}^{2}\right) \\
& \Rightarrow 2(1-\alpha) \sigma_{1}^{2}+2 \rho(1-\alpha) \sigma_{1} \sigma_{2}-2 \rho \alpha \sigma_{1} \sigma_{2}+2 \alpha \sigma_{2}^{2}
\end{aligned}
$$

\subsection{Mathematical Formulation and Analysis for the n-Assets MPT Model}

Reference [14] MPT is the theory that tries to minimize risk of the return for a certain level of expected return hence its model can be written as

$$
\begin{gathered}
\text { min imize } \frac{1}{2} \sum_{i=1}^{n} \sum_{j=1}^{n} \sigma_{i j} X_{i} X_{j} \\
\text { subject to } \sum_{i=1}^{n} X_{i} \mu_{i}=\mu_{p} \\
\text { and } \sum_{i=1}^{n} X_{i}=1
\end{gathered}
$$

Which means find the portfolio strategy that minimizes the portfolio variance $\sigma_{p}{ }^{2}$ for a given target of expected rate of the return of the portfolio $\mu_{p}$,

\subsection{The Solution to This $N$-Asset Model Will be as Follows}

For the sake of simplifying the calculations this equation can be changed to the matrix form and it can be written as

$$
\begin{array}{ll}
\text { minimize } & Z=\frac{1}{2} X^{T} V X \\
\text { subject to } & S=X \in \Re^{n} \mid X^{T} \mu=\mu_{p} \\
& X^{T} e=1
\end{array}
$$

Where $X=\left[x_{1}, x_{2}, x_{3}, \ldots x_{n}\right]^{T}$ is the column vector of portfolio weights for each security

$V$-is the covariance matrix of the return

$e=[1,1,1,1, . . ., .1]^{T} e \in \mathbb{R}^{n}$

$\mu_{p}$ is the desired level of the expected return of the portfolio

We have to note that in this model formulation

1. The admissible set includes short selling i.e. portfolio positions with negative weights $\left(X_{i}<0\right)$ are allowed

2. The parameter $\mu_{p}$ is exogenous given

3. The model equation (1) is a convex quadratic programming problem i.e. the objective function is quadratic with the linear constraints and the feasible set $\mathrm{S}$ is convex

4. The solution of the model depends on the parameter $\mu_{p}$ To avoid complications we impose some conditions that

(i) random variables are twice differentiable (ii) no two securities can have the same expected return hence vectors $e, \mu$ are linearly independent, this can be proved by using the real data from DSE

(iii) The covariance matrix is strictly positive definite. The positivity of the covariance matrix means that all the n-assets are indeed risky, and this is the case of our portfolio since we consider stocks only.

In which we found that the model problem (5) is a convex quadratic problem with a unique convex solution because the function $X^{T} V X$ defines a quadratic function and matrix $\mathrm{V}$ is symmetric and positive definite as from condition (iii)

The constraints are linear which shows that $\mathrm{S}$ is a convex solution space. Also condition (ii) shows that the gradient of the constraints are linearly independent which guarantee a unique solution. Therefore, since conditions (i), (ii) and (iii) hold then the model problem (5) has a unique solution and well formulated.

\subsection{Solution to this Equation}

After applying the Langrangian method to the equations

$$
\begin{aligned}
& f(x)=\frac{1}{2} X^{T} V X, \quad \mathrm{~g}(\mathrm{x})=\left(g_{1}, g_{2}\right)^{T} \\
& g_{1}(x)=X^{T} e-1=0, \mathrm{~g}_{2}(x)=X^{T} \mu-\mu_{p}=0
\end{aligned}
$$

The model equation becomes

$$
\begin{aligned}
& \text { min imize } \mathrm{z}=\frac{1}{2} X^{T} V X \\
& \text { subjectto } \mathrm{g}(\mathrm{x})=\left(\begin{array}{c}
X^{T} e-1 \\
X^{T} \mu-\mu_{p}
\end{array}\right)=\overrightarrow{0}
\end{aligned}
$$

and the resulting langrangian equation for the portfolio model becomes $\quad L(X, \lambda)=\frac{1}{2} X^{T} V X-\lambda_{1}\left(X^{T} e-1\right)-\lambda_{2}\left(X^{T} \mu-\mu_{p}\right) \quad$ from which the optimal point $X^{*}$ was found to be

$$
X^{*}=V^{-1}\left(\lambda_{1} e+\lambda_{2} \mu\right)
$$

and $\mu_{p}=\mu^{T} V^{-1} V X^{*}$ where $\lambda_{1}=\frac{c-\mu_{p} b}{a c-b^{2}}$ and $\lambda_{2}=\frac{a \mu_{p}-b}{a c-b^{2}}$

And it is noted that $\lambda_{1}$ and $\lambda_{2}$ depends on $\mu_{p}$ which is the target portfolio mean approved in the variance minimization problem. The variables a, $\mathrm{b}$, and $\mathrm{c}$ can be calculated because $V$ and $\mu$ are known and therefore $X^{*}=V^{-1}\left(\lambda_{1} e+\lambda_{2} \mu\right)$ can be solved to get $X^{*}=\left[X_{1}{ }^{*} X_{2}{ }^{*} X_{3}{ }^{*} \ldots X_{n}{ }^{*}\right]^{T}$ as the optimal portfolio weight.

\subsection{Variance $\sigma_{p}{ }^{*^{2}}$ for the Optimal Portfolio $\left(X^{*}\right)$}

The portfolio variance is given by $\sigma_{p}{ }^{2}=X^{T} V X$ and the optimal portfolio variance was found from the equation 
$\sigma_{p}^{*^{2}}=X^{*^{T}} V X^{*} \quad$ from which after solving we get $\sigma_{p}^{*^{2}}=\lambda_{1}+\lambda_{2} \mu_{p} \quad$ substituting the value of $\lambda_{1}$ and $\lambda_{2}$ it results to $\sigma_{p}^{*^{2}}=\frac{c-\mu_{p} b}{a c-b^{2}}+\frac{a \mu_{p}-b}{a c-b^{2}} \mu_{p}$ after simplifying we get

$$
\sigma_{p}^{*^{2}}=\frac{a \mu_{p}^{2}-2 b \mu_{p}+c}{d} \text { where } d=a c-b^{2}
$$

When $\sigma_{p}^{*^{2}}$ is plotted against $\mu_{p}$ for the equation (6) the set of the minimum variance portfolio is a parabola and when $\mu_{p}$ is plotted against $\sigma_{p}^{*^{2}}$ the set of the minimum variance portfolio is a hyperbola called the frontier which has two part the efficient and the inefficient part the upper being called the efficient frontier and the lower being called the inefficient frontier. Note that for all portfolios on the efficient frontier there is no other portfolio with the same mean and a lower standard deviation (risk). Also for each inefficient portfolio there is an efficient portfolio with the same variance but a higher expected rate of return

Technically we defined the efficient frontier, inefficient frontier and the frontier as

\subsection{The Restricted Mean - Variance Model}

In the preceding section we have explicitly demonstrated the solution methods of the unrestricted mean - variance model. In this classical Markowitz model, the investment is unrestricted. That is for example there are no short - sale restrictions no diversification restrictions and no costs constraints. And therefore this makes the model more theoretical than practical. For more details about criticism on the classical Markowitz model read [14] in [16] and [17]. However in the real life investment problems, restrictions are often imposed either by regulatory authorities as policy, some restrictions are due to financial constraints or limitations, some are internal restrictions in the company which must be observed, and many more other forms of restrictions exist

These restrictions in an investment model should be incorporated in regard to specific investment environment. The danger arises if and when an investment model meant for a certain environment is used to a new environment without any modification. It is therefore against this background that in this chapter we modify the preceding classical Markowitz model by imposing some diversification restrictions, so as to reflect the DSE investment environment.

Forms of diversification Restrictions in investment

Reference [14] the forms of diversification are;-

1. Percentage amount of a portfolio invested in a stock, this means some investors can limit their portfolios to constitute a certain amount of stocks and no more stocks could be added to that portfolio.

2. Financial or liquidity restrictions, these are usually very important for commercial banks say a certain amount of money is availed to invest in stock and not more can be invested.

3. Pre - specified bounds within which the fractions of wealth invested in the securities must lie, in this restriction there is a fixed amount of wealth that is allowed to be invested in securities and normally these are set by the companies themselves.

4. Number of securities to constitute the investment portfolio, here there are some of the investors limit the number of stocks to constitute their portfolio and no more stocks could be added to the portfolio if the limited number is reached. However for our model we shall consider diversification in the form of 2, 3, and 4 in which cases.

For diversification 2, we shall ensure that all the available wealth is to be invested in stocks. That is it is the sum that has been designated for investment in stocks. Therefore we are not interested in the other securities in the firm's portfolio, so to us, the only portfolio that exists consists of stocks only.

In consideration of stock restriction 3 we shall require that our portfolio weight $X=\left[X_{1}, X_{2}, \ldots \mathrm{X}_{n}\right]^{T}$ have a lower bound of ' $a$ ' and an upper bound ' $b$ ', that is $a \leq X \leq b, \mathrm{a}, \mathrm{b} \in \mathbb{R}^{n}$

For diversification restriction 4 , we shall restrict the minimum number of stocks available from which to form an investment portfolio. Say a maximum of 8 stocks and, a minimum of 2 stocks.

These restrictions have great implications on both mathematical and economic point of view and in this work we will base on the mathematical part and we are going to find the mathematical implications of 3 and 4 restrictions above.

\subsection{Mathematical Implication of Diversification Restriction Four}

In consideration of diversification constraint 4 we try to find out mathematically the effect of increasing or reducing the number of stocks held in a portfolio. In the frontier, we examined the necessary and sufficient conditions for a security to improve the Markowitz hyperbola (frontier). let $P_{1}=\left\{S_{1}, S_{2} \ldots S_{n}\right\}$ be a set of $n$ securities among which we may choose for our portfolio, $P_{2}=P_{1} \mid\left\{S_{i}\right\}=\left\{S_{1} S_{2} \ldots S_{i-1} S_{i+1} \ldots S_{n}\right\}$ and let $\psi_{p}$ and $\psi_{q}$ be the Markowitz hyperbolas for securities set $P_{1}$ and $P_{2}$ respectively.

[14] a unique portfolio weights $X^{*}=g+h \mu_{p}$ can be determined for securities that lie on the hyperbola as a linear function of the portfolio expected return $\mu_{p}$, where $g$ and $h$ are known constants for a particular portfolio. And from $X^{*}=V^{-1}\left(\lambda_{1} e+\lambda_{2} \mu\right)$ for the unrestricted model problem where $\lambda_{1}=\frac{c-\mu_{p} b}{a c-b^{2}}$ and $\lambda_{2}=\frac{a \mu_{p}-b}{a c-b^{2}}$ substituting for $\lambda_{1}$ and $\lambda_{2}$ gives $X^{*}=V^{-1}\left(\left(\frac{c-b \mu_{p}}{d}\right) e+\left(\frac{a \mu_{p}-b}{d}\right) \mu\right)$ after 
simplifying we get

$$
\Rightarrow X^{*}=\frac{\left(c V^{-1} e-b V^{-1} \mu\right)}{d}+\frac{\left(a V^{-1} \mu-b V^{-1} e\right)}{d} \mu_{p}
$$

And hence

$$
X^{*}=g+h \mu_{p}
$$

where $g=\frac{\left(c V^{-1} e-b V^{-1} \mu\right)}{d}$ and $h=\frac{\left(a V^{-1} \mu-b V^{-1} e\right)}{d}$

Then from the equation $X^{*}=g+h \mu_{p}$ we see that

i. If $g_{i}=h_{i}=0$ then $\psi_{p}=\psi_{q}$

ii. If $g_{i} \neq 0$ and $h_{i}=0$ then $\psi_{p} \neq \psi_{q}$ and any point on

$\psi_{p}$ has a non-zero fixed weight of the $i^{\text {th }}$ security

iii.If $h_{i} \neq 0$ then $\psi_{p} \neq \psi_{q}$ and hence $\psi_{p}$ and $\psi_{q}$ are tangents at exactly 1 point.

Corollary 1

$$
g_{i}=h_{i}=0 \text { if } \psi_{p}=\psi_{q}
$$

\section{Proof}

Suppose $g_{i}=h_{i}=0$ does not hold, that is $(a) h_{i}=0$ and $g_{i} \neq 0$, or $(b.) h_{i} \neq 0$ in part $(b.) g_{i}$ is not conditioned because if $h_{i} \neq 0$ whether $g_{i}=0$, or $g_{i} \neq 0$ the effect of the $\mathrm{i}^{\text {th }}$ security carries the same mathematical implication on $\psi_{p}$

From theorem part (ii) if $h_{i}=0$ and $g_{i} \neq 0$ then $\psi_{p} \neq \psi_{q}$ which contradicts (a.). Also from theorem part (iii) if $h_{i} \neq 0$ then $\psi_{p} \neq \psi_{q}$ this contradicts (b.) Therefore, we conclude that $\psi_{p}=\psi_{q}$ implying $g_{i}=h_{i}=0$. But from theorem part (ii) if $g_{i}=h_{i}=0$, then $\psi_{p}=\psi_{q}$ hence $g_{i}=h_{i}=0$ if $\psi_{p}=\psi_{q}$

As from [14] the following theorems and its proofs holds Theorem 1

$$
g_{i}=h_{i}=0 \text { if }\left(V^{-1} e\right)_{i}=\left(V^{-1} \mu\right)_{i}=0
$$

Proof

Assume $g_{i}=h_{i}=0$ then from the equation 3 we have $c\left(V^{-1} e\right)_{i}=b\left(V^{-1} \mu\right)_{i}$ and $b\left(V^{-1} e\right)_{i}=a\left(V^{-1} \mu\right)_{i}$ then from $c\left(V^{-1} e\right)_{i}=b\left(V^{-1} \mu\right)_{i}$ we get

$$
\left(V^{-1} e\right)_{i}=\frac{b}{c}\left(V^{-1} \mu\right)_{i}
$$

and from $b\left(V^{-1} e\right)_{i}=a\left(V^{-1} \mu\right)_{i}$ we have

$$
\left(V^{-1} e\right)_{i}=\frac{a}{b}\left(V^{-1} \mu\right)_{i}
$$

combining equations (7) and (7.1) we get $\frac{b}{c}\left(V^{-1} \mu\right)_{i}=\frac{a}{b}\left(V^{-1} \mu\right)_{i} \quad$ simplifying this results to the equation

$$
a\left(V^{-1} \mu\right)_{i}=\frac{b^{2}}{c}\left(V^{-1} \mu\right)_{i}
$$

In equation (7) we can see that if $\left(V^{-1} \mu\right)_{i} \neq 0$ then we conclude $a=\frac{b^{2}}{c} \quad$ which means $a c=b^{2} \quad$ and $d=a c-b^{2}=0$ which is impossible, because in the proof claim 1 we proved that $d=a c-b^{2}>0$ so $\left(V^{-1} \mu\right)_{i}=0$

Since $c\left(V^{-1} e\right)_{i}=b\left(V^{-1} \mu\right)_{i}$ and $\left(V^{-1} \mu\right)_{i}=0$ it implies that also $c\left(V^{-1} e\right)_{i}=0$ but $\mathrm{c}>0$ therefore $\left(V^{-1} e\right)_{i}=0$ hence $g_{i}=h_{i}=0$ if $\left(V^{-1} e\right)_{i}=\left(V^{-1} \mu\right)_{i}=0$

Conversely

Assume $\quad\left(V^{-1} e\right)_{i}=\left(V^{-1} \mu\right)_{i}=0 \quad$ then clearly $\frac{1}{d} c\left(V^{-1} e\right)_{i}-b\left(V^{-1} \mu\right)_{i}=0 \quad$ but $\quad g_{i}=\frac{1}{d} c\left(V^{-1} e\right)_{i}-b\left(V^{-1} \mu\right)_{i}=0$ hence $g_{i}=0$ and $\frac{1}{d}\left(a\left(V^{-1} \mu\right)_{i}-b\left(V^{-1} e\right)_{i}\right)=0$ but again $h_{i}=\frac{1}{d}\left(a\left(V^{-1} \mu\right)_{i}-b\left(V^{-1} e\right)_{i}\right)=0 \quad$ implying $\quad h_{i}=0$, thus $\left(V^{-1} e\right)_{i}=\left(V^{-1} \mu\right)_{i}=0$ which implies $g_{i}=h_{i}=0$

Corollary 2

$$
\left(V^{-1} e\right)_{i}=\left(V^{-1} \mu\right)_{i}=0 \text { if } \psi_{p}=\psi_{q}
$$

\section{Proof}

From corollary 1 we have $g_{i}=h_{i}=0$ if $\psi_{p}=\psi_{q}$ also from theorem 2 we have $g_{i}=h_{i}=0$ if $\left(V^{-1} e\right)_{i}=\left(V^{-1} \mu\right)_{i}=0$ which means that both theorem 2 and corollary 1 implies $\left(V^{-1} e\right)_{i}=\left(V^{-1} \mu\right)_{i}=0$ if $\psi_{p}=\psi_{q}$ and corollary 2 is the final result that we were intending to prove. This provides a necessary and sufficient condition for some security $S_{n+1}$ to improve the Markowitz hyperbola. This will be so provided the addition of $S_{n+1}$ to the existing security set, $P=\left\{S_{1}, S_{2}, \ldots S_{n}\right\}$ in such that the new covariance matrix 
$V_{n e w}$ which includes $S_{n+1}$ is invertible and the conditions $\left(V_{n e w} \mu\right)_{n+1}=\left(V_{n e w} e\right)_{n+1}=0$ does not hold.

Diversification restriction 4

Imposing the restriction $a \leq X \leq b \mid a b X \in \mathbb{R}^{n}$ where in general we assume that $\sum_{i=1}^{n} a_{i} \leq 1, \sum_{j=1}^{n} b_{j} \geq 1$ hold.

Not that $\sum_{i=1}^{n} a_{i} \leq 1$ is necessary for the portfolio optimization problem to have a solution and $\sum_{j=1}^{n} b_{j} \geq 1$ assures us that the total wealth available will be invested. And hence our optimization problem 1 will be equal to that in [14] which is

$$
\begin{aligned}
\min \text { imize } \quad Z_{r} & =\frac{1}{2} X^{T} V X \\
\text { subjectto } S r & =\left\{X \in \mathbb{R}^{n} \mid X^{T} \mu=\mu_{p},\right. \\
X^{T} e & \left.=1, a \leq X \leq b \mid a, b, X \in \mathbb{R}^{n}\right\}
\end{aligned}
$$

To be more specific this requires the weights to be nonnegative $(X \geq 0)$. Therefore, we restrict $a=0$ so that we have $0 \leq X \leq b$ and our problem becomes

$$
\begin{array}{cl}
\min \text { imize } & Z_{r}=\frac{1}{2} X^{T} V X \\
\text { subjectto } & S r=\left\{X \in \mathbb{R}^{n} \mid X^{T} \mu=\mu_{p},\right. \\
& X^{T} e=1, X \geq 0, \\
& \left.X \leq b \mid 0, b, X \in \mathbb{R}^{n}\right\}
\end{array}
$$

This equation has to be written as a quadratic programming problem in the form

$$
\begin{aligned}
& \min \text { imize }(\max \text { imize }) Z=c X+X^{T} D X \\
& \text { subject to } A X \leq b, X \geq 0
\end{aligned}
$$

Where $\quad X=\left(x_{1}, x_{2}, \ldots x_{n}\right)^{T}, c=\left(c_{1}, c_{2}, \ldots c_{n}\right)^{T}, b=\left(b_{1}, b_{2}, \ldots b_{n}\right)^{T} \quad A$ and $D$ are $n x n$ matrices and $X^{T} D X$ defines a quadratic form. The matrix $\mathrm{D}$ is assumed symmetric and positive definite for minimization problem and symmetric negative definite maximization problem. The constraints are linear which guarantee a convex solution space.

Hence the optimization problem (3.10) can be formulated as a quadratic programming problem is

$$
\begin{aligned}
& \text { min imize } Z=\frac{1}{2} X^{T} V X \\
& \text { subjectto }_{r}=\left\{I X \leq b, X \geq 0, X^{T} \mu=\mu_{p}, X^{T} e=1\right\}
\end{aligned}
$$

Where

$$
X=\left(x_{1}, x_{2}, \ldots x_{n}\right)^{T}, \mu=\left(\mu_{1}, \mu_{2}, \ldots \mu_{n}\right)^{T}, e=(1,1, \ldots 1)^{T}, \quad I \text { is }
$$

an $n x n$ identity matrix and $V$ is an $n x n$ covariance matrix.
Combining the inequality constraints $I X \leq b$ and $(X \geq 0)$ the model problem can be written as minimize $Z=\frac{1}{2} X^{T} V X$

$$
\text { subjectto } \mathrm{G}_{1}(\mathrm{X})=X^{T} e-1=0, \mathrm{G}_{2}(\mathrm{X})=X^{T} \mu-\mu_{p}=0
$$

$G_{3}(X)=\left(\begin{array}{c}1 \\ -1\end{array}\right) X-\left(\begin{array}{l}b \\ 0\end{array}\right) \leq 0$ and hence the model has to be solved in this form.

$\lambda_{s}$ start at $\lambda_{3}$ because $\lambda_{1}$ and $\lambda_{2}$ are used in the unrestricted model problem with linear constraints.

As in [14] applying the KKT conditions on the inequality constraints it yield

$$
\begin{gathered}
\lambda_{b} \leq 0, \lambda_{a} \leq 0 \\
\nabla Z-\left(\lambda^{T}{ }_{b}, \lambda^{T}{ }_{a}\right) \nabla G X=0 \\
\lambda_{b j}\left(b_{i}-X_{i}\right)=0 \\
\lambda_{a k} X_{i}=0 \mid k=n+3, n+4 \ldots 2 n+2, \\
j=3,4, \ldots n+2, i=1,2, \ldots n \\
1 X \leq b \\
-X \leq 0 \\
\nabla Z=X^{T} V \text { and } \nabla \mathrm{G}(\mathrm{X})=\left(\begin{array}{c}
I \\
-I
\end{array}\right)
\end{gathered}
$$

Substituting

$$
\nabla Z=X^{T} V \text { and } \nabla \mathrm{G}(\mathrm{X})=\left(\begin{array}{c}
I \\
-I
\end{array}\right)
$$
into

$\nabla Z-\left(\lambda^{T}{ }_{b}, \lambda^{T}{ }_{a}\right) \nabla G X=0$ it gives $X^{T} V+\left(\lambda^{T}{ }_{a}-\lambda^{T}{ }_{b}\right) I=0$

Let $S=b-I X \geq 0$ be the slack variables of the constraints. Then the KKT reduce to

$$
\begin{aligned}
& X^{T} V+\left(\lambda^{T}{ }_{a}-\lambda^{T}{ }_{b}\right) I=0 \\
& I X+S=b \\
& \lambda_{a k} X_{i}=0=\lambda_{b j} S_{j} \mid k=n+3, n+4 \ldots 2 n+2, \\
& j=3,4, \ldots n+2, i=1,2, \ldots n \\
& \lambda_{a}, \lambda_{b}, X, S \geq 0
\end{aligned}
$$

It is easily seen that the inequality constraints have been converted into the equality constraints using the KKT conditions. Therefore the general restricted model equation from which we can find the solution is

$$
\begin{aligned}
& X^{T} V-\lambda_{1} e-\lambda e \mu+\left(\lambda^{T}{ }_{a}-\lambda^{T}{ }_{b}\right) I=0 \\
& I X+S=b \\
& X^{T} e=1 \\
& X^{T} \mu=\mu_{p} \\
& \lambda_{a k} X_{i}=0=\lambda_{b j} S_{j} \mid k=n+3, n+4 \ldots 2 n+2, \\
& j=3,4, \ldots n+2, i=1,2, \ldots n \\
& \lambda_{a}, \lambda_{b}, X, S \geq 0
\end{aligned}
$$


We have to understand that two types of risk exist namely the systematic risk and unsystematic risk. Diversification on its part generally does not protect against systematic risk because a drop in the entire market and economy typically affects all investments. But in real sense diversification is designed to decrease unsystematic risk. Since unsystematic risk is the possibility that one single item or one single thing will deviate from the normal risk and return at a certain time, having a portfolio invested in a variety of stocks, a variety of asset classes and a variety of sectors will lower the risk of losing much money when one investment type declines in value.

\section{Results and Discussion}

The data was obtained at the Dar-Es-Salaam Stock exchange market (DSE) offices and there are about 17 registered stocks namely Tanzania Breweries Limited (TBL) Swissport Tanzania Limited (Swissport), TOL Gases Limited (TOL), Tanzania Portland Cement Company Limited (TWIGA), TATEPA LIMITED, Kenya Airways Limited (KA), Tanzania Cigarette Company (TCC), East African Breweries Limited (EABL), Tanga Cement Company Limited (SIMBA), Jubilee Holdings Limited (JHL), Dar Es Salaam Community Bank (DCB),CRDB Bank PLC (CRDB), National Microfinance Bank (NMB), Kenya Commercial Bank (KCB). Eleven stocks were selected to represent the DSE market. And from these eleven stocks the general trend of the DSE market was studied.

The data was collected on $17^{\text {th }}$ of April 2014, includes the closing prices, opening prices, trading dates, market capitalization, high and low prices, turnover and company names. The data provided was for nine years from 2006 to 2014. From the data it was observed that seven companies started selling their stocks in 2006, three companies started on 2007, three companies on 2008, one company on 2009, another one on 2011 and the other two companies on 2013. Also it was observed that some of the companies have sold their stocks ones, twice or thrice which it is difficult to get the real trend for these data. Even though we were provided all these data only the company names, closing dates and trading dates was employed in the calculations and its outputs were used in plotting the relevant graphs. To calculate the monthly returns the formula

$$
R_{i}=\frac{\text { clossing price-previous closing price }}{\text { previous closing price }}
$$

Was used, and to get the portfolio mean we used the formulae

$$
\mu_{p}=\sum_{i=1}^{n} X_{i} \mu_{i}
$$

The correlation and covariance matrices calculated are as in the table 1 below. And for the case of optimal constrained and un-constrained frontier their outcome are as in the tables 2 and table 3 and the corresponding frontier are as in the Figure 1 and figure 2 respectively. From these graphs we can found that the unconstrained and the constrained frontier provides nearly the same optimal return but the unconstrained having the greater change as you go up or down from the optimal point, this is of course the expected output because constraints affects the performance of the portfolio.

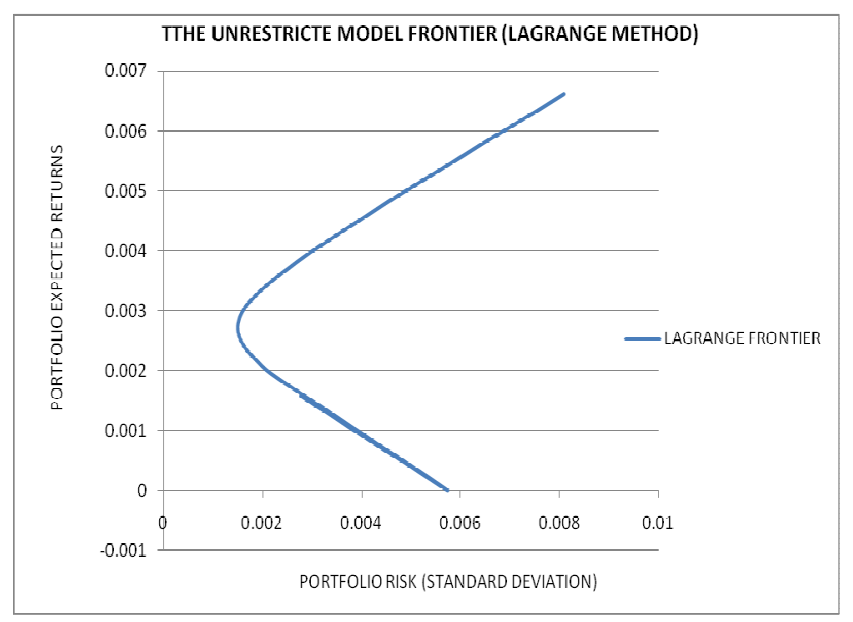

Fig 1. The DSE Unrestricted model frontier

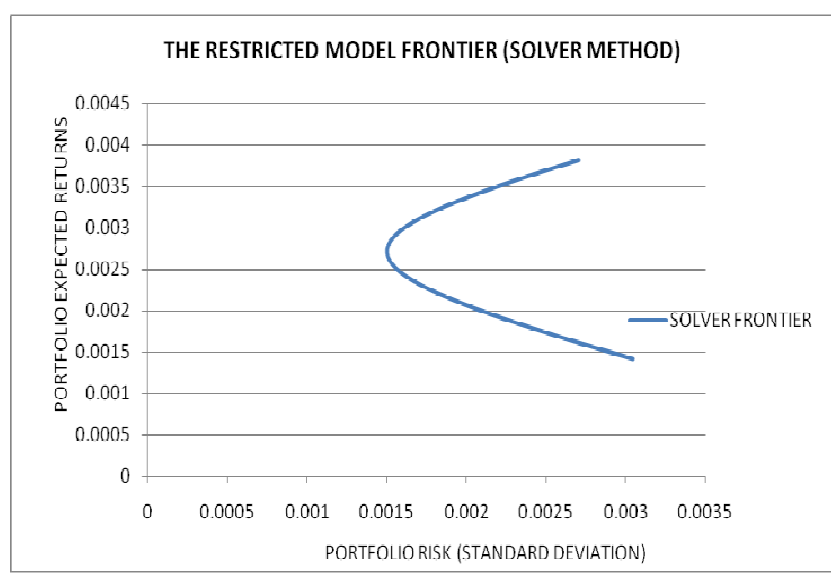

Fig 2. The DSE Restricted model frontier

For the case of diversification to improve in the existing portfolio Markowitz requires that assets must be negatively correlated. To prove this two pairs of stocks in our portfolio were chosen, where the first pair is for the most negatively correlated stocks and the second represents the most positively correlated stocks, as we can read it from our covariance matrix The terms most positively and most negatively correlated stocks refers only to this portfolio of 11 stocks and for the data used as from 2006 to April 2014 so it should not be taken as the general conclusion for any portfolio since portfolio change over time due to its stochastic distribution. Moreover, the most correlated stocks today may change and become the most negatively correlated stocks in future.

To see how these stocks behave in relation to each other we plotted the constrained frontier for the NMB and TTCL as the most positively stocks as in the figure 3 where we have observed that the portfolio seem to be improving by 
minimizing risk to a certain level and increasing the return on the upper part of the hyperbola called the efficient frontier.

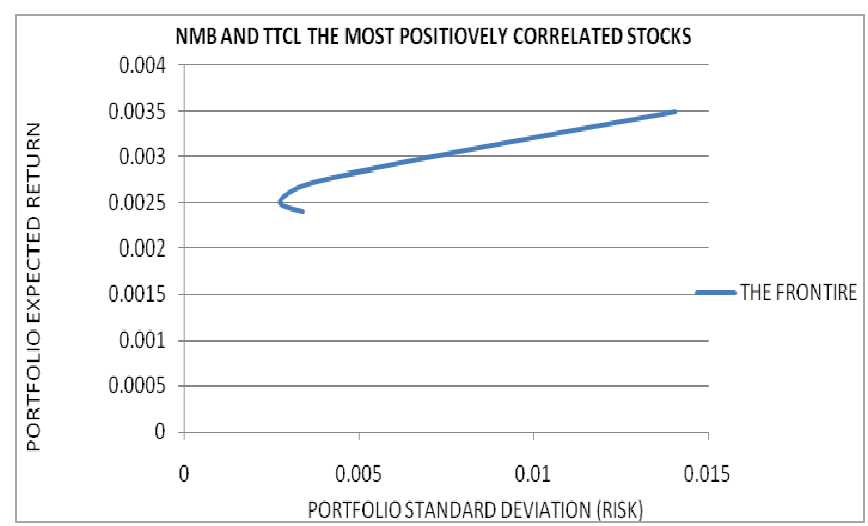

Figure 3. The NMB and TTCL the most positively correlated stocks

On the other hand we explored the idea of diversification by adding the new stocks to the existing portfolio and we realized that the new portfolio with additional of stocks has a greater return than the first one as seen in figure 4 where the diversification was basing on the stock correlation variation.

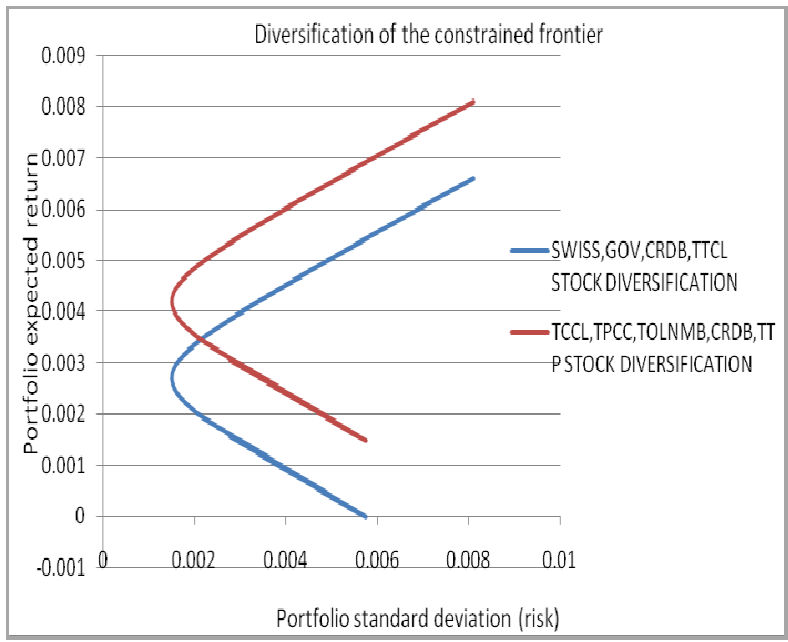

Fig 4. The DSE Diversification of constrained frontier

using the condition we proved in corollary 2 of Chapter $3 \mathrm{pg}$ 10 , which is independent of the correlation of the assets; the condition only required us to compute the new covariance matrix $V_{\text {new }}$ that includes the additional stock and then check if $V_{n e w}^{-1} \mu=V^{-1}{ }_{n e w} e=0$ does not hold, since when this condition does not hold then the new stock added will improve the frontier. We started with a portfolio of five stocks which are SWISS, TCCL, TBL, DCB, and GOV with covariance matrix $V$, then we added a new stock and computed the new covariance matrix $V_{\text {new }}$ and $V_{\text {new }}^{-1}$ checked the condition and found that; $V^{-1}{ }_{n e w} \mu \neq V^{-1}{ }_{n e w} e \neq 0$, We keep on adding the new stocks to the existing one and compute the new covariance matrix and its inverse but the results were still the same that $V_{n e w}^{-1} \mu \neq V_{\text {new }}^{-1} e \neq 0$ showing that the additional of the new stock to the existing portfolio increases the performance of the portfolio. And when we plotted their frontiers, we observed that the frontier for the portfolio with many stocks was above the one with fewer stocks

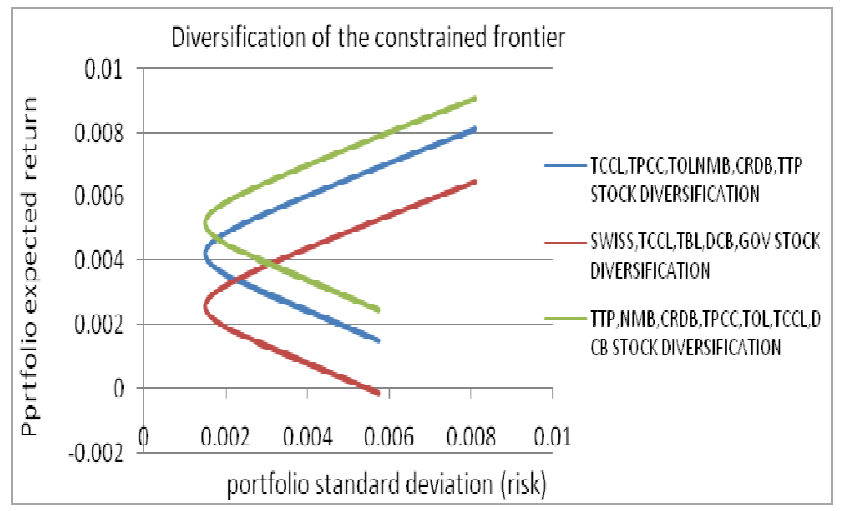

Fig 5. The DSE diversification of the constrained frontier

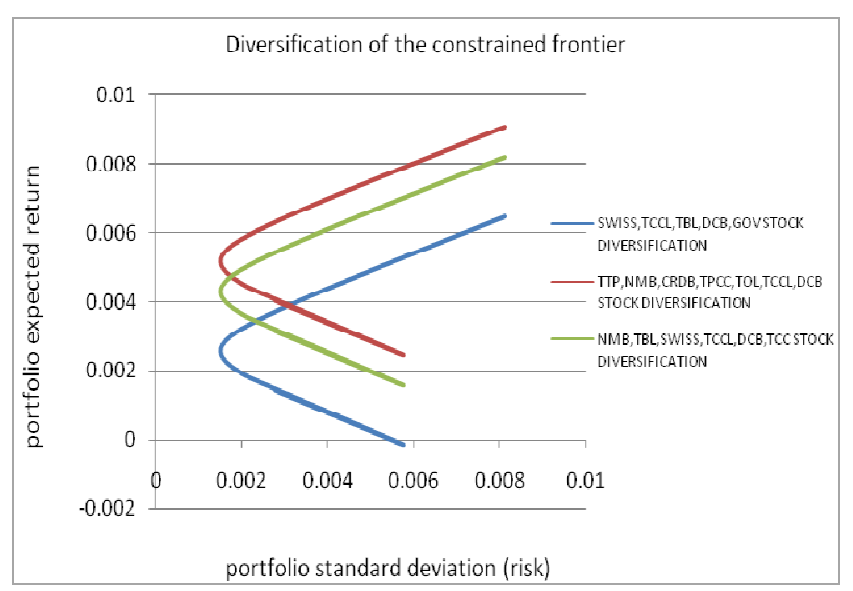

Fig 6. The DSE Diversification of the constrained frontier

Graphs in figure 5 and figure 6 shows the diversification of the constrained frontier in the way that as you increase the stocks in the existing portfolio the portfolio return increases, this is preferably because as you increase stocks to a portfolio the risk level is reduced even though this is not always the case because sometimes it may be constant but with lower risk.

Moreover it is seen that, even if the investor own the portfolio with stocks only he or she can still improve it by diversifying. On other hand investors who prefer lower returns with known risks than higher returns with unknown risks can still invest and reduce the risk by diversifying into different DSE stocks. 
Table 1. The correlation and covariance matrix for the DSE stocks.

\begin{tabular}{|c|c|c|c|c|c|c|c|c|c|c|c|}
\hline \multicolumn{12}{|c|}{ Portfolio Optimization Model (11 Risky Assets) Solver Method } \\
\hline & & & & & $\begin{array}{l}\text { Assets } \\
\text { Data } \\
\end{array}$ & $\begin{array}{l}\text { Expected } \\
\text { return }\end{array}$ & $\begin{array}{l}\text { Standard } \\
\text { Deviation }\end{array}$ & & & & \\
\hline & & & & & SWISS & 0.00095 & 0.0039 & & & & \\
\hline & & & & & NMB & 0.000646 & 0.0033 & & & & \\
\hline & & & & & GOV & -0.057545 & 0.2743 & & & & \\
\hline & & & & & DCB & $9.9 \mathrm{E}-05$ & 0.0072 & & & & \\
\hline & & & & & CRDB & $5.9 \mathrm{E}-05$ & 0.0053 & & & & \\
\hline & & & & & TTP & -0.06193 & 0.2254 & & & & \\
\hline & & & & & ТРCC & 0.000953 & 0.0033 & & & & \\
\hline & & & & & TOL & 0.002351 & 0.0174 & & & & \\
\hline & & & & & TCC & 0.003599 & 0.0061 & & & & \\
\hline & & & & & TBL & -0.145596 & 0.6847 & & & & \\
\hline & & & & & TCCL & -0.000857 & 0.0139 & & & & \\
\hline \multicolumn{12}{|c|}{ CORRELATION MATRIX FOR THE DSE STOCKS } \\
\hline & SWISS & NMB & GOV & DCB & CRDB & TTP & ТPCC & TOL & $\mathrm{TCC}$ & TBL & TCCL \\
\hline SWISS & 1 & 0.1106 & -0.02479 & -0.05878 & 0.067013 & -0.1441 & -0.24011 & -0.1977 & 0.004756 & 0.0863 & 0.05277 \\
\hline NMB & 0.110567 & 1 & -0.1156 & -0.05878 & 0.1429274 & 0.38516 & 0.232902 & -0.1977 & 0.148742 & -0.114 & -0.4558 \\
\hline GOV & -0.024794 & -0.116 & 1 & 0.01113 & -0.029931 & -0.1519 & -0.03615 & 0.09137 & -0.04915 & -0.032 & 0.04938 \\
\hline DCB & -0.058777 & -0.059 & 0.01113 & 1 & 0.2794789 & 0.21856 & -0.2778 & -0.0964 & -0.05537 & 0.0029 & 0.0832 \\
\hline CRDB & 0.067013 & 0.1429 & -0.02993 & 0.27948 & 1 & 0.3135 & -0.06657 & 0.00322 & 0.178728 & 0.162 & -0.0317 \\
\hline TTP & -0.14413 & 0.3852 & -0.15185 & 0.21856 & 0.3134961 & 1 & 0.199989 & 0.13826 & -0.00157 & 0.0308 & -0.0116 \\
\hline ТPCC & -0.240107 & 0.2329 & -0.03615 & -0.2778 & -0.066573 & 0.19999 & 1 & 0.16946 & -0.00157 & 0.0061 & -0.0296 \\
\hline TOL & -0.197686 & -0.198 & 0.09137 & -0.09643 & 0.0032195 & 0.13826 & 0.169461 & 1 & -0.03514 & 0.212 & -0.1053 \\
\hline TCC & 0.004756 & 0.1487 & -0.04915 & -0.05537 & 0.1787278 & -0.1665 & -0.00157 & -0.0351 & 1 & -0.067 & -0.0176 \\
\hline TBL & 0.086283 & -0.114 & -0.03159 & 0.00289 & 0.1619936 & 0.03082 & 0.006149 & 0.21202 & -0.06674 & 1 & -0.0247 \\
\hline TCCL & 0.052773 & -0.456 & 0.04938 & 0.0832 & -0.031732 & -0.0116 & -0.02958 & -0.1053 & -0.01756 & -0.025 & 1 \\
\hline \multicolumn{12}{|c|}{ THE COVARIANCE MATRIX FOR THE DSE MARKET } \\
\hline & SWISS & NMB & GOV & DCB & CRDB & TTP & TPCC & TOL & $\mathrm{TCC}$ & TBL & TCCL \\
\hline SWISS & $9.22 \mathrm{E}-06$ & $1 \mathrm{E}-06$ & $-4.9 \mathrm{E}-05$ & $-1 \mathrm{E}-06$ & $-3.23 \mathrm{E}-07$ & $-4 \mathrm{E}-05$ & $-9.1 \mathrm{E}-07$ & $-5 \mathrm{E}-06$ & $8.66 \mathrm{E}-08$ & 0.0001 & $2.2 \mathrm{E}-06$ \\
\hline NMB & $1.04 \mathrm{E}-06$ & $1 \mathrm{E}-05$ & -0.00011 & $-1.1 \mathrm{E}-07$ & 2.307E-06 & 0.00017 & $2.58 \mathrm{E}-06$ & 8.9E-09 & 3.04E-06 & $-3 \mathrm{E}-04$ & $-2 \mathrm{E}-05$ \\
\hline GOV & $-4.85 \mathrm{E}-05$ & $-1 \mathrm{E}-04$ & 0.08055 & $5.1 \mathrm{E}-06$ & $-5.23 \mathrm{E}-05$ & -0.0018 & $-2.2 \mathrm{E}-05$ & $6 \mathrm{E}-05$ & $-8.4 \mathrm{E}-05$ & -0.007 & 0.00019 \\
\hline DCB & $-1.01 \mathrm{E}-06$ & $-1 \mathrm{E}-07$ & $5.1 \mathrm{E}-06$ & $3.7 \mathrm{E}-05$ & $6.079 \mathrm{E}-06$ & 0.00011 & $-5.3 \mathrm{E}-06$ & $-4 \mathrm{E}-06$ & $-1.4 \mathrm{E}-06$ & $1 \mathrm{E}-05$ & $2.5 \mathrm{E}-06$ \\
\hline CRDB & $-3.23 \mathrm{E}-07$ & 2E-06 & $-5.2 \mathrm{E}-05$ & $6.1 \mathrm{E}-06$ & $2.745 \mathrm{E}-05$ & 0.00015 & $-8.9 \mathrm{E}-07$ & $-1 \mathrm{E}-06$ & $5.62 \mathrm{E}-06$ & 0.0006 & $-2 \mathrm{E}-06$ \\
\hline ТTP & $-4.2 \mathrm{E}-05$ & 0.0002 & -0.00181 & 0.00011 & 0.0001495 & 0.02258 & $7.94 \mathrm{E}-05$ & 0.00017 & $3.31 \mathrm{E}-05$ & -0.005 & $-7 \mathrm{E}-05$ \\
\hline TPCC & $-9.05 \mathrm{E}-07$ & $3 \mathrm{E}-06$ & $-2.2 \mathrm{E}-05$ & $-5.3 \mathrm{E}-06$ & $-8.93 \mathrm{E}-07$ & 7.9E-05 & $1.02 \mathrm{E}-05$ & $5.1 \mathrm{E}-06$ & $-3 \mathrm{E}-08$ & $6 \mathrm{E}-05$ & $-1 \mathrm{E}-06$ \\
\hline TOL & $-4.68 \mathrm{E}-06$ & 9E-09 & $6 \mathrm{E}-05$ & $-3.6 \mathrm{E}-06$ & $-1.11 \mathrm{E}-06$ & 0.00017 & $5.13 \mathrm{E}-06$ & 0.00011 & $-3.7 \mathrm{E}-06$ & 0.0002 & $-2 \mathrm{E}-06$ \\
\hline $\mathrm{TCC}$ & $8.66 \mathrm{E}-08$ & $3 \mathrm{E}-06$ & $-8.4 \mathrm{E}-05$ & $-1.4 \mathrm{E}-06$ & 5.619E-06 & 3.3E-05 & $-3 \mathrm{E}-08$ & $-4 \mathrm{E}-06$ & 3.6E-05 & $-3 \mathrm{E}-04$ & $-1 \mathrm{E}-06$ \\
\hline TBL & 0.000146 & $-3 \mathrm{E}-04$ & -0.0074 & $1.1 \mathrm{E}-05$ & 0.000619 & -0.0046 & $6.06 \mathrm{E}-05$ & 0.00015 & -0.00029 & 0.5257 & -0.0003 \\
\hline TCCL & $2.16 \mathrm{E}-06$ & $-2 \mathrm{E}-05$ & 0.00019 & $2.5 \mathrm{E}-06$ & $-2.43 \mathrm{E}-06$ & $-7 \mathrm{E}-05$ & $-1.3 \mathrm{E}-06$ & $-2 \mathrm{E}-06$ & $-1.5 \mathrm{E}-06$ & $-3 \mathrm{E}-04$ & 0.0002 \\
\hline
\end{tabular}


Table 2. Table for the unconstrained frontier

\begin{tabular}{|c|c|c|c|c|c|c|c|c|c|c|c|c|}
\hline \multicolumn{13}{|c|}{ Generating The Un-Constrained Frontier (Lagrange Method) } \\
\hline \multirow{2}{*}{$\begin{array}{l}\text { Optimal } \\
\text { Standard } \\
\text { Deviation } \\
\end{array}$} & \multirow{2}{*}{$\begin{array}{l}\text { Optimal } \\
\text { Portfolio } \\
\text { Returns } \\
\end{array}$} & \multicolumn{11}{|c|}{ Corresponding Optimal Portfolio Weights } \\
\hline & & SWISS & NMB & GOV & DCB & CRDB & TTP & TPCC & TOL & TCC & TBL & TCCL \\
\hline \multirow[t]{2}{*}{0.00343967} & 0.0012 & 0.092308 & 0.092308 & 0.0923 & 0.09231 & 0.092308 & 0.092307692 & 0.092307692 & -0.83077 & 0.092308 & 0.092308 & 0.092308 \\
\hline & & 0 & 0 & 0 & 0 & 0 & 0 & 0 & 0 & 0 & 0 & 0 \\
\hline 0.00277385 & 0.001573 & 0.121025 & 0.121025 & 0.121 & 0.12102 & 0.121025 & 0.12102462 & 0.121025 & -1.08922 & 0.121025 & 0.121025 & 0.121025 \\
\hline 0.00574509 & $-1.3 \mathrm{E}-09$ & $-1 \mathrm{E}-07$ & $-1 \mathrm{E}-07$ & $-1 \mathrm{E}-07$ & $-1 \mathrm{E}-07$ & $-1 \mathrm{E}-07$ & $-9.995 \mathrm{E}-08$ & $-1 \mathrm{E}-07$ & $9 \mathrm{E}-07$ & $-1 \mathrm{E}-07$ & $-1 E-07$ & $-1 E-07$ \\
\hline 0.00217403 & 0.001946 & -0.17763 & 0.116229 & 0.1315 & 0.11285 & 0.129806 & 0.12302518 & 0.119627 & 0.133205 & 0.114546 & 0.099269 & 0.09757 \\
\hline 0.00191419 & 0.002134 & -0.07801 & 0.106833 & 0.1165 & 0.1047 & 0.115395 & 0.11111929 & 0.108976 & 0.11754 & 0.105772 & 0.096137 & 0.095066 \\
\hline 0.00170451 & 0.002321 & 0.020561 & 0.097537 & 0.1016 & 0.09664 & 0.101138 & 0.09933961 & 0.098438 & 0.10204 & 0.097091 & 0.093039 & 0.092588 \\
\hline 0.00156173 & 0.002507 & 0.118599 & 0.088291 & 0.0868 & 0.08862 & 0.086957 & 0.08762304 & 0.087957 & 0.086624 & 0.088456 & 0.089957 & 0.090124 \\
\hline 0.0015033 & 0.002694 & 0.217693 & 0.078945 & 0.0718 & 0.08052 & 0.072623 & 0.07578025 & 0.077363 & 0.071042 & 0.079728 & 0.086841 & 0.087633 \\
\hline 0.0015401 & 0.00288 & 0.315731 & 0.069699 & 0.057 & 0.0725 & 0.058442 & 0.06406368 & 0.066881 & 0.055626 & 0.071094 & 0.083759 & 0.085168 \\
\hline 0.00166654 & 0.003068 & 0.414825 & 0.060353 & 0.0421 & 0.0644 & 0.044108 & 0.0522209 & 0.056287 & 0.040043 & 0.062366 & 0.080644 & 0.082677 \\
\hline 0.00186216 & 0.003253 & 0.512864 & 0.051107 & 0.0273 & 0.05638 & 0.029927 & 0.04050432 & 0.045805 & 0.024627 & 0.053732 & 0.077562 & 0.080213 \\
\hline 0.00210988 & 0.00344 & 0.61143 & 0.041811 & 0.0124 & 0.04832 & 0.01567 & 0.02872465 & 0.035268 & 0.009128 & 0.045051 & 0.074463 & 0.077735 \\
\hline 0.00239455 & 0.003628 & 0.710524 & 0.032465 & -0.0026 & 0.04022 & 0.001336 & 0.01688186 & 0.024673 & -0.00645 & 0.036323 & 0.071348 & 0.075244 \\
\hline 0.0027003 & 0.003813 & 0.808562 & 0.023219 & -0.0174 & 0.0322 & -0.012845 & 0.00516529 & 0.014192 & -0.02187 & 0.027689 & 0.068266 & 0.072779 \\
\hline 0.00302452 & 0.004 & 0.907128 & 0.013923 & -0.0322 & 0.02414 & -0.027102 & -0.0066144 & 0.003654 & -0.03737 & 0.019008 & 0.065167 & 0.070302 \\
\hline 0.00336256 & 0.004188 & 1 & 0.007333 & -0.0484 & 0.01703 & -0.042457 & -0.0186748 & -0.00676 & -0.05437 & 0.011066 & 0.064646 & 0.070606 \\
\hline 0.00370754 & 0.004374 & 1 & 0.041705 & -0.0826 & 0.0246 & -0.072876 & -0.0339026 & -0.01437 & -0.09241 & 0.014837 & 0.102643 & 0.11241 \\
\hline 0.00405902 & 0.004561 & 1 & 0.076076 & -0.1169 & 0.03218 & -0.103295 & -0.0491304 & -0.02198 & -0.13044 & 0.018608 & 0.14064 & 0.154214 \\
\hline 0.00441352 & 0.004747 & 1 & 0.110263 & -0.1509 & 0.03972 & -0.133551 & -0.0642766 & -0.02956 & -0.16827 & 0.022358 & 0.178433 & 0.195794 \\
\hline 0.00477377 & 0.004933 & 1 & 0.144634 & -0.1851 & 0.0473 & -0.163971 & -0.0795044 & -0.03717 & -0.2063 & 0.026129 & 0.21643 & 0.237597 \\
\hline 0.005139 & 0.005121 & 1 & 0.17919 & -0.2195 & 0.05491 & -0.194553 & -0.0948138 & -0.04483 & -0.24454 & 0.02992 & 0.254631 & 0.279625 \\
\hline 0.00550472 & 0.005308 & 1 & 0.213561 & -0.2538 & 0.06249 & -0.224972 & -0.1100415 & -0.05244 & -0.28257 & 0.033691 & 0.292628 & 0.321429 \\
\hline 0.00587241 & 0.005494 & 1 & 0.247932 & -0.288 & 0.07007 & -0.255392 & -0.1252693 & -0.06005 & -0.32061 & 0.037461 & 0.330625 & 0.363233 \\
\hline 0.00623976 & 0.00568 & 1 & 0.282119 & -0.322 & 0.07761 & -0.285648 & -0.1404155 & -0.06763 & -0.35844 & 0.041212 & 0.368418 & 0.404813 \\
\hline 0.00661242 & 0.005868 & 1 & 0.316675 & -0.3565 & 0.08522 & -0.31623 & -0.1557249 & -0.07528 & -0.39667 & 0.045003 & 0.406619 & 0.446841 \\
\hline 0.00698224 & 0.006053 & 1 & 0.350862 & -0.3905 & 0.09276 & -0.346487 & -0.1708711 & -0.08285 & -0.4345 & 0.048753 & 0.444412 & 0.488421 \\
\hline 0.00735502 & 0.00624 & 1 & 0.385233 & -0.4247 & 0.10034 & -0.376906 & -0.1860989 & -0.09047 & -0.47254 & 0.052524 & 0.482409 & 0.530225 \\
\hline 0.00772863 & 0.006426 & 1 & 0.419605 & -0.4589 & 0.10792 & -0.407325 & -0.2013267 & -0.09808 & -0.51057 & 0.056295 & 0.520406 & 0.572029 \\
\hline 0.00810295 & 0.006613 & 1 & 0.453976 & -0.4932 & 0.11549 & -0.437745 & -0.2165545 & -0.1057 & -0.5486 & 0.060065 & 0.558403 & 0.613832 \\
\hline
\end{tabular}

Table 3. Table for the constrained frontier

\begin{tabular}{|c|c|c|c|c|c|c|c|c|c|c|c|c|}
\hline \multicolumn{13}{|c|}{ Generating The Constrained Frontier (Solver Method) } \\
\hline \multirow{2}{*}{$\begin{array}{l}\text { Optimal } \\
\text { Standard } \\
\text { Deviation } \\
\end{array}$} & \multirow{2}{*}{$\begin{array}{l}\text { Optimal } \\
\text { Portfolio } \\
\text { Returns } \\
\end{array}$} & \multicolumn{11}{|c|}{ Corresponding Optimal Portfolio Weights } \\
\hline & & SWISS & NMB & GOV & DCB & CRDB & TTP & TPCC & TOL & TCC & TBL & TCCL \\
\hline 0.0026956 & 0.001619 & $-1 \mathrm{E}-06$ & 0 & 0.3991 & 0 & 0.167824 & 0.12472471 & 0.0531 & 0.229449 & 0.025813 & 0 & 0 \\
\hline 0.00304132 & 0.00142 & 0 & 0 & 0.2027 & 0 & 0 & 0 & 0 & 0.797274 & 0 & 0 & 0 \\
\hline 0.00277385 & 0.001573 & 0.021417 & 0.008035 & 0.109 & 0.00981 & 0.000894 & 0.00446036 & 0.006248 & 0.79638 & 0.008921 & 0.016956 & 0.01785 \\
\hline 0.0025455 & 0.001709 & 0.095204 & 0 & 0.0937 & 0.00153 & 0 & 0 & 0 & 0.780453 & 0 & 0.013772 & 0.015304 \\
\hline 0.00217256 & 0.001947 & 0.206818 & 0 & 0.0482 & 0 & 0 & 0 & 0 & 0.732998 & 0 & 0.004285 & 0.007718 \\
\hline 0.00191545 & 0.002133 & 0.291456 & 0 & 0.0118 & 0 & 0 & 0 & 0 & 0.695092 & 0 & 0 & 0.001658 \\
\hline 0.00170547 & 0.00232 & 0.367984 & 0 & 0 & 0 & 0 & 0 & 0 & 0.632016 & 0 & 0 & 0 \\
\hline 0.00156173 & 0.002507 & 0.442648 & 0 & 0 & 0 & 0 & 0 & 0 & 0.557352 & 0 & 0 & 0 \\
\hline 0.00150336 & 0.002693 & 0.517312 & 0 & 0 & 0 & 0 & 0 & 0 & 0.482688 & 0 & 0 & 0 \\
\hline 0.00154055 & 0.002881 & 0.592376 & 0 & 0 & 0 & 0 & 0 & 0 & 0.407624 & 0 & 0 & 0 \\
\hline 0.00166566 & 0.003067 & 0.66664 & 0 & 0 & 0 & 0 & 0 & 0 & 0.33336 & 0 & 0 & 0 \\
\hline 0.00186216 & 0.003253 & 0.741304 & 0 & 0 & 0 & 0 & 0 & 0 & 0.258696 & 0 & 0 & 0 \\
\hline 0.00210988 & 0.00344 & 0.815968 & 0 & 0 & 0 & 0 & 0 & 0 & 0.184032 & 0 & 0 & 0 \\
\hline 0.00239296 & 0.003627 & 0.890632 & 0 & 0 & 0 & 0 & 0 & 0 & 0.109368 & 0 & 0 & 0 \\
\hline 0.002702 & 0.003814 & 0.965696 & 0 & 0 & 0 & 0 & 0 & 0 & 0.034304 & 0 & 0 & 0 \\
\hline 0.00284924 & 0.0039 & 1 & 0 & 0 & 0 & 0 & 0 & 0 & 0 & 0 & 0 & 0 \\
\hline
\end{tabular}

\section{Conclusions}

The study has explored the DSE historical stocks daily trading data for the last nine years (from November 2006 to April 2014). The correlation and covariance matrices were calculated and used in calculating the optimal portfolio returns with minimum risks. It is observed that diversification is important for the investors risk reduction mechanism and the paper encourage investors to diversify their portfolio. Moreover it is observed that the DSE stocks form a stochastic 
market, because stocks have a random relationship and the stock return vary over time either can increase or decrease depending on the market conditions, as realized from their correlation matrix. The Mathematical and theoretical implications of the classical Modern portfolio theory (MPT) model was explored and its arguments were tested against the DSE stocks data where it was realized that the DSE data analysis concur with the theory that, when two negatively correlated assets are combined they reduce risk more than when positively correlated assets are combined. More over it was realized that this theory works even if the stocks are not absolutely negatively correlated. In addition to that it was found that when the graphs of returns against standard deviation (risk) are plotted for both the restricted and unrestricted optimization problem we get the hyperbola, and the portfolio can be improved by adding more stocks to the existing one. Finally we encourage individuals of all economic levels to invest in stocks of the Dar es Salaam stock exchange market so as to improve investments and increase their future return.

\section{Acknowledgements}

I would like to express my sincere gratitude to the Nelson Mandela Institution of Science and Technology for financial assistance and the Rungwe District Council for granting me a study leave.

\section{References}

[1] Gary C, and Uri G, (2010), portfolio choice and risk attitudes: an experiment, Economic Inquiry (ISSN 0095-2583), Vol. 48, No. 1, January 2010, $133-146$

[2] Robert J. Barro (1990), the review of financial studies, department of Economics, Harvard University, Littauer 120, Cambridge, MA 02138

[3] Darrel, D. and singleton K, (2003) Credit Risk: Pricing Measurement and Management, Sci.Aging knowl.Environ 2003(44), or 23

[4] Edwin J. Elton and Martin J. Gruber, "Modern portfolio theory, 1950 to date", Journal of Banking \& Finance 21 (1997)
1743-1759

[5] Markowitz, H. 1952, 'Portfolio Selection,' Journal of Finance, 7, 77-99. Efficient frontiers using estimated parameters. Ann. Operations

[6] Sharpe,W, F, (1964) Capital asset prices: A theory of market equilibrium under conditions of risk." Journal of Finance 19 (3): $425-442$.

[7] Craig W. Hoden (2008), Excel modelling and Estimation in Investment (third Edition), Prentice Hall inc, upper saddle River. New Jersey 07458

[8] Ziemba, W. T., (1993), the effect of errors in means, variances and covariance on optimal portfolio choice, $J$. Portfolio Management, (Winter) 6-11

[9] Tobin, James. (1958a) Liquidity Preference as Behavior towards Risk. Review of Economic Studies 25 (2): 65-86

[10] Broadie, M, (1993), Computing efficient frontiers using estimated parameters, Ann, Operations Research 45 (1): 21-58

[11] Konno, H., (1988), Portfolio Optimization using LI Risk Function IHSS Report 88-9, Inst. of Human and Social Sciences, Tokyo Institute of Technology.

[12] Puelz, A.V, (2002) A Stochastic Convergence Model for Portfolio Selection." Operations Research 50 (3): 462-476.

[13] Konno, H, Yamazaki, H, (1991), Mean-Absolute Deviation Portfolio Optimization Model and Its Applications to Tokyo Stock Market." Management Science 37 (5): 519-531.

[14] F. Mayanja, S. Mataramvura and W. Charles, (2011), "A Mathematical Approach to a Stocks Portfolio Selection: The Case of Uganda Securities Exchange (USE)," Journal of Mathematical Finance, Vol. 3 No. 4, 2013, pp. 487-501. doi: 10.4236/jmf.2013.34051.

[15] Nitis Mukhopadyyay, (2000), Probability and Statistics Inference. University of Connecticut Storrs, Connecticut. Marcel Dekker, Inc.

[16] Vern Sumnicht, (2008) Practical Applications of Post-Modern Portfolio Theory.

[17] Loeper B. David, (2000) Modern Portfolio Reality (MPR).The Failures of Modern Portfolio Theory", Finance ware, Inc. 